\title{
New Accurate and Efficient Discrete Formulation for Reduced Tracer Aggregation Population Balance Equation
}

\author{
Mehakpreet Singh ${ }^{1}$, Vivek Ranade ${ }^{1}$, and Gavin Walker ${ }^{1}$ \\ ${ }^{1}$ University of Limerick
}

July 11, 2020

\begin{abstract}
In this work, a new discrete formulation for a tracer aggregation population balance equation is developed based on an assumption that the particles properties are concentrated on the representative of the cell. The new formulation is simpler, easy to code and can be easily adaptable to any kind of grid. The proposed method is extended to solve a mixed-suspension mixed-product removal (MSMPR) system in which aggregation and nucleation are main mechanisms that effect the particles properties. The performance of the new formulation is tested against the cell average technique (Giri and Nagar, ESAIM-Math. Model. Num., 49(2), 349-372 (doi.org/10.1051/m2an/2014035)) for different analytically tractable problems. The testing is conducted by taking into consideration two bench marking cases, namely, batch and MSMPR systems using both gelling and non gelling kernels. It is demonstrated that the new scheme is computationally less expensive and predicts the numerical results with high precision even on a coarser grid. For even a refined grid, the new scheme tends to produce better results than the cell average technique at a lesser computational time.
\end{abstract}

\section{Hosted file}

Manuscript.pdf available at https://authorea.com/users/341916/articles/468799-new-accurateand-efficient-discrete-formulation-for-reduced-tracer-aggregation-population-balanceequation 
figures/cell-discet/cell-discet-eps-converted-to.pdf 
figures/cell1/cell1-eps-converted-to.pdf 
figures/sum-3-0/sum-3-0-eps-converted-to.pdf 
figures/sum-2-0/sum-2-0-eps-converted-to.pdf 
figures/sum-1-0/sum-1-0-eps-converted-to.pdf 
figures/sum-3-I/sum-3-I-eps-converted-to.pdf 
figures/sum-2-I/sum-2-I-eps-converted-to.pdf 
figures/sum-1-I/sum-1-I-eps-converted-to.pdf 
figures/sum-3-I-5/sum-3-I-5-eps-converted-to.pdf 
figures/sum-2-I-5/sum-2-I-5-eps-converted-to.pdf 
figures/sum-1-I-5/sum-1-I-5-eps-converted-to.pdf 
figures/prod-3-0/prod-3-0-eps-converted-to.pdf 
figures/prod-2-0/prod-2-0-eps-converted-to.pdf 
figures/prod-1-0/prod-1-0-eps-converted-to.pdf 
figures/prod-3-I-5/prod-3-I-5-eps-converted-to.pdf 
figures/prod-2-I-5/prod-2-I-5-eps-converted-to.pdf 
figures/prod-1-I-5/prod-1-I-5-eps-converted-to.pdf 
figures/sum-1-M-0/sum-1-M-0-eps-converted-to.pdf 
figures/sum-2-M-0/sum-2-M-0-eps-converted-to.pdf 
figures/sum-1-M-I/sum-1-M-I-eps-converted-to.pdf 
figures/sum-2-M-I/sum-2-M-I-eps-converted-to.pdf 
figures/prod-1-M-0/prod-1-M-0-eps-converted-to.pdf 
figures/prod-2-M-0/prod-2-M-0-eps-converted-to.pdf 
figures/prod-1-M-I/prod-1-M-I-eps-converted-to.pdf 
figures/prod-2-M-I/prod-2-M-I-eps-converted-to.pdf 